\title{
The effects of temporal delay and orientation on haptic object recognition
}

\author{
MatT CRAdDock ANd Rebecca LaWson \\ University of Liverpool, Liverpool, England
}

\begin{abstract}
We examined the effects of interstimulus interval (ISI) and orientation changes on the haptic recognition of novel objects, using a sequential shape-matching task. The stimuli consisted of 36 wedge-shaped plastic objects that varied along two shape dimensions (hole/bump and dip/ridge). Two objects were presented at either the same orientation or a different orientation, separated by either a short (3-sec) ISI or a long (15-sec) ISI. In separate conditions, ISI was blocked or randomly intermixed. Participants ignored orientation changes and matched on shape alone. Although performance was better in the mixed condition, there were no other differences between conditions. There was no decline in performance at the long ISI. There were similar, marginally significant benefits to same-orientation matching for short and long ISIs. The results suggest that the perceptual object representations activated from haptic inputs are both stable, being maintained for at least $15 \mathrm{sec}$, and orientation sensitive.
\end{abstract}

Comparing objects haptically is something we do more often than we might think. Suppose, late at night, you arrive home and start to search for your front door key. It's too dark to see clearly, so you feel through the keys on your key ring and try a likely one. If it isn't the right one, you keep searching, trying to remember which keys you have already felt and the shape of the key that you are searching for. How effectively can we maintain these haptic memories of object shape over time?

One efficient solution for achieving such a goal might be for the haptic system to share the processes available for visual object recognition. Indeed, in many everyday situations, information from both modalities is combined to guide our actions, particularly when our goals require manipulating objects.

Converging evidence from a variety of methodologies has revealed broad similarities between vision and haptics. There is substantial overlap in the neural architecture invoked during visual and haptic object exploration (e.g., Amedi, Jacobson, Hendler, Malach, \& Zohary, 2002; Amedi, Malach, Hendler, Peled, \& Zohary, 2001; Miquée et al., 2008), and visual and haptic object recognition are similarly impaired by changes in object orientation (Craddock \& Lawson, 2008; Lacey, Peters, \& Sathian, 2007; Lawson, 1999, 2009; Newell, Ernst, Tjan, \& Bülthoff, 2001) and object size (Craddock \& Lawson, 2009a, 2009b).

Nevertheless, inputs from vision and haptics are not equivalent and must first traverse separate pathways; each modality will be subject to different limitations even if, ultimately, they share common perceptual representations. Although haptic object recognition is reasonably fast (e.g., Craddock \& Lawson, 2008, 2009a; Klatzky,
Lederman, \& Metzger, 1985), it is generally slower than vision (e.g., Craddock \& Lawson, 2009a). Haptics relies on slower, more sequential exploration than does vision and must, therefore, depend more on working and shortterm memory to maintain and integrate information as it accumulates. The haptic object-processing system should, therefore, be optimized for storing input over many seconds and, so, may be less sensitive to temporal delays than is vision. This was tested in a sequential object-matching study that varied interstimulus interval (ISI).

Previous studies examining the influence of ISI on tactile and haptic memory present somewhat mixed evidence. Nevertheless, there appears to be an important distinction between those experiments examining passive touch and those examining active, haptic exploration. Studies employing passive, tactile tasks reveal steadily declining memory as ISI increases. Three studies testing recall of the location of a tactile stimulus applied to the forearm after delays of 0-60 sec (Gilson \& Baddeley, 1969; Miles \& Borthwick, 1996; Sullivan \& Turvey, 1972) reported worse performance as delay increased. Similarly, Gallace, Tan, Haggard, and Spence (2008) found that memory for tactile stimuli applied to multiple locations on the body simultaneously was worse at longer stimulus-probe delays. However, it is unclear what results from such tasks imply for haptic recognition and matching of actively explored complex 3-D shapes.

Three studies have reported worse performance on a haptic 3-D shape-matching performance over time, consistent with the tactile memory studies discussed above. Millar (1974) tested children matching novel shapes with delays of 5-30 sec. Matching was worse as delays increased. Kiphart, Hughes, Simmons, and Cross (1992)

M. Craddock, m.craddock@liv.ac.uk 
reported that matching of LEGO block objects was equally good for 5- and 15-sec ISIs but was much worse after 30- and 45-sec ISIs. Finally, Woods, O'Modhrain, and Newell (2004) tested matching of L-shaped stimuli. Relative to 0 -sec delays, matching was worse for 15 - and 30 -sec delays.

These results contrast with those reported in a fourth study. Norman, Clayton, Norman, and Crabtree (2008) tested matching of plastic molds of bell peppers. Hit rates improved as ISI increased from 3 to 9 to $15 \mathrm{sec}$, and response bias declined as ISI increased: After $3 \mathrm{sec}$, participants were strongly biased to respond same, but this bias was almost eliminated after $15 \mathrm{sec}$. Norman et al. argued that longer ISIs facilitated the encoding and consolidation of memory for object shape.

Increasing ISI would usually be expected to make performance worse in any modality, since transient representations are no longer available and information must, instead, be retained by an imperfect memory system. However, the mixed findings in the studies reviewed above suggest that this may not be the case for haptics. Although passive tactile studies (Gallace et al., 2008; Gilson \& Baddeley, 1969; Miles \& Borthwick, 1996; Sullivan \& Turvey, 1972) have consistently pointed to a simple sensory trace that decays over time, active haptic studies have reported performance improving (Norman et al., 2008), as well as deteriorating (Kiphart et al., 1992; Millar, 1974; Woods et al., 2004), during the seconds following haptic exploration.

One way to track changes in how information is stored over time is to measure sensitivity to task-irrelevant perceptual information. Previous research has shown that haptic object recognition suffers when there is a task-irrelevant change of object orientation between study and subsequent recognition (Craddock \& Lawson, 2008; Lacey et al., 2007; Lawson, 2009; Newell et al., 2001), similar to the orientation sensitivity observed in visual object recognition (e.g., Lawson, 1999; Tarr \& Cheng, 2003). Furthermore, several studies have demonstrated that visual orientation sensitivity generally declines with increasing ISIs (Ellis \& Allport, 1986; Ellis, Allport, Humphreys, \& Collis, 1989; Humphrey \& Lupker, 1993; Lawson \& Humphreys, 1996). This weakening of orientation sensitivity may indicate a shift from perceptual to more abstract representations and a change from viewer-centered to object-centered representations. If vision and haptics share comparable memorial processes, haptic object recognition may also show declining orientation sensitivity at longer ISIs. Alternatively, if haptics is optimized for maintaining stable perceptual representations, it may not show such a decline.

We reported a same-orientation benefit in a haptic old-new recognition task across delays of around $15 \mathrm{~min}$ (Craddock \& Lawson, 2008) and at short intervals of about $5 \mathrm{sec}$ in a sequential-matching task (Lawson, 2009). However, we are not aware of any studies that have compared orientation sensitivity across different delays. Thus, the evidence reviewed above leaves open two important questions: whether ISI influences haptic object recognition and whether ISI modulates haptic orientation sensitivity.

In the present study, participants performed a sequential haptic object-matching task with ISIs of 3 and $15 \mathrm{sec}$

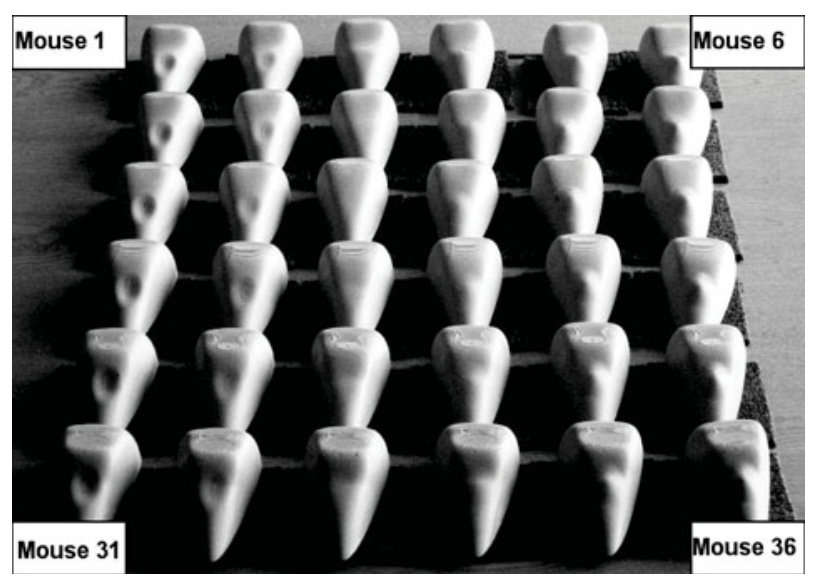

Figure 1. The "mice" all facing forward and arranged in their $6 \times 6$ shape space. The $x$-dimension, which varies the shape of the front of each mouse, runs from the left column (holes) to the right column (bumps) in the figure; the $y$-dimension, which varies the shape of the rear of each mouse, runs from the back row (dips) to the front row (ridges) in the figure.

for pairs of objects either presented at the same orientation or rotated by $90^{\circ}$ in depth from each other. Since we were interested in the nature of the perceptual representations used in the task, we used novel objects that all shared the same basic shape and, so, were difficult to distinguish either semantically or verbally.

Participants received either a single, randomly mixed block of trials at both ISIs or two blocks of trials, one with a 3 -sec ISI and another with a 15 -sec ISI. In the mixed condition, unlike in the blocked condition, the participants could not predict the ISI on each trial and, thus, could not easily use different strategies for each ISI. As such, there may be less effect of ISI in the mixed condition.

\section{METHOD}

\section{Participants}

Sixty-four participants (50 of them female) from the University of Liverpool were recruited in return for course credit. In both the blocked and the mixed conditions, 27 participants were right-handed and 5 left-handed. Ages ranged from 18 to 57 years $(M=21)$.

\section{Stimuli}

The stimuli were 36 rigid plastic "mice" printed using a Dimension 3D ABS plastic printer. They varied parametrically on two dimensions, each with six levels, giving a $6 \times 6$ shape space (see Figure 1). Stimuli were numbered from 1 at the top left (deepest hole and deepest dip; see Figure 2A) to 6 at the top right (tallest bump and deepest dip; see Figure 2B) to 31 at the bottom left (deepest hole and tallest ridge; see Figure 2C) and 36 at the bottom right (tallest bump and tallest ridge; see Figure 2D).

Each mouse was fixed to a $10-\mathrm{cm}$-square base. The experimenter put the mice into a square hole in the apparatus depicted in Figure 3. One side of the hole was marked with green tape, and an adjacent side with red tape. The front tip of the mouse was aligned with either the green or the red tape for green or red orientations, respectively, so there was a $90^{\circ}$ depth rotation on orientation change trials.

The participants sat facing the apparatus. The participants' right hands entered a $12-\mathrm{cm}$-square aperture in order to touch the mouse (Figure 3), breaking an infrared beam that shone across this aperture. The time that the beam was broken was recorded as the onset of 
A

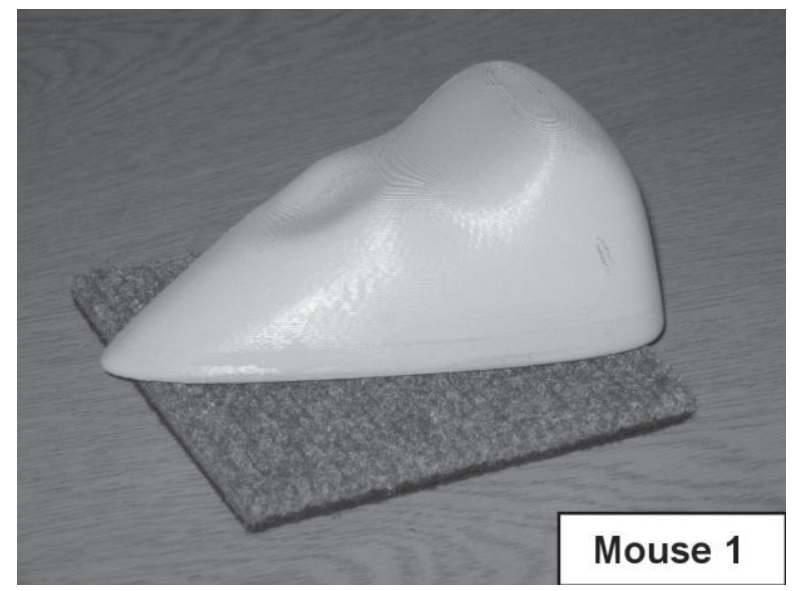

C

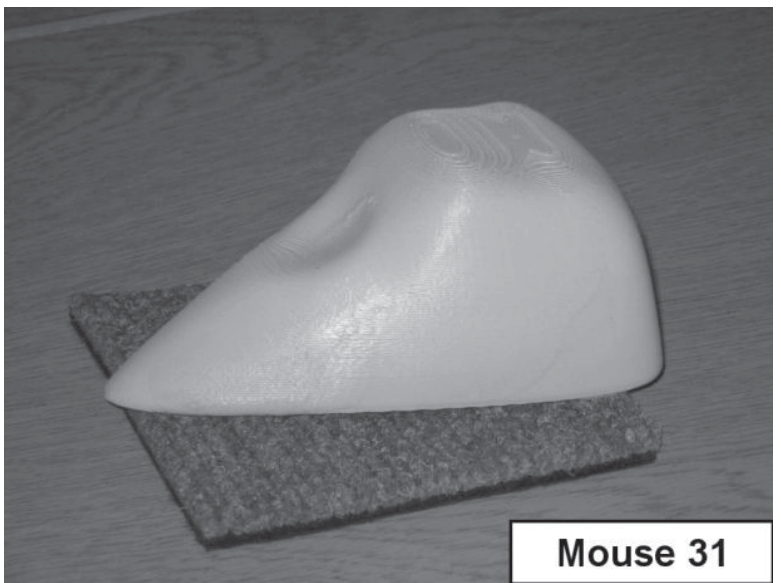

B

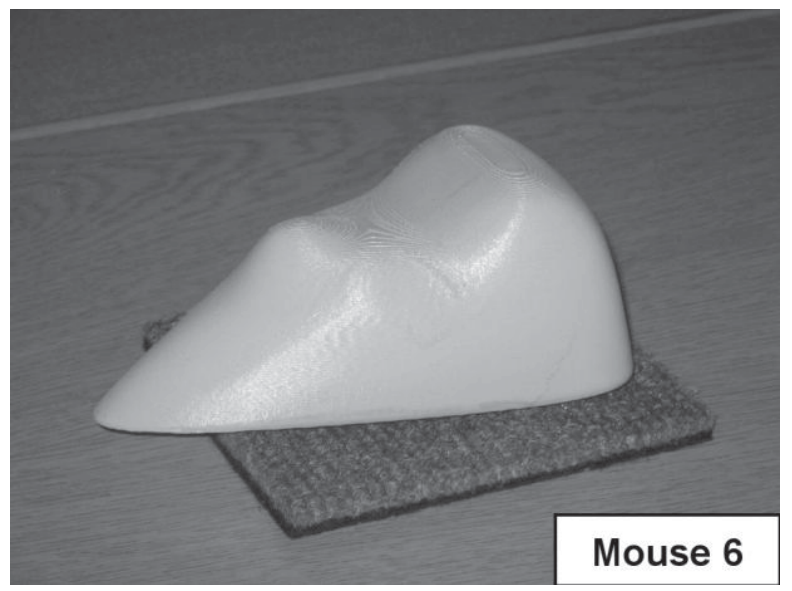

D

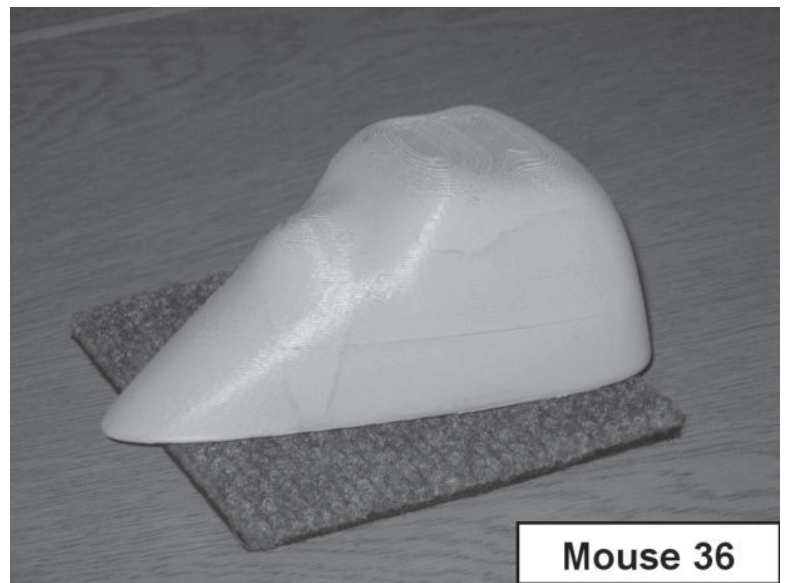

Figure 2. Side views of the four mice at each corner of the shape space.

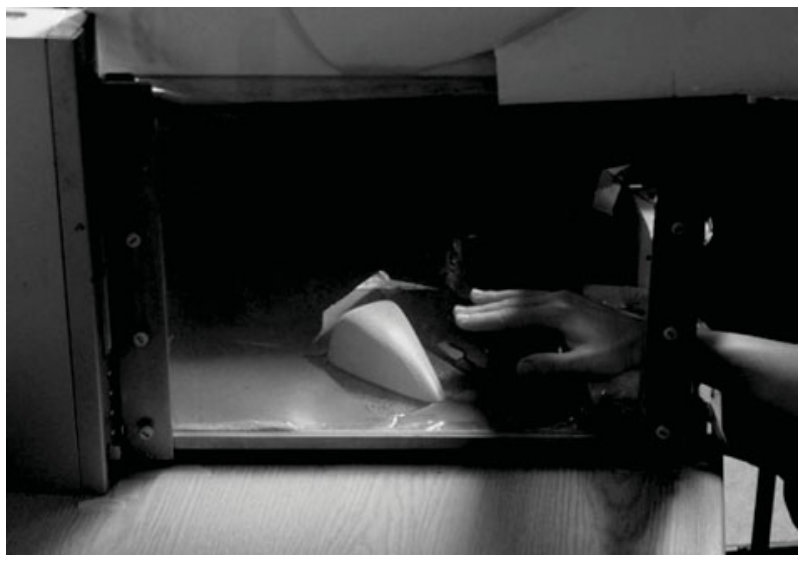

Figure 3. Mouse 15 in the red orientation with a participant reaching to touch it with the right hand. The mouse was rotated by $90^{\circ}$ counterclockwise from this position in the green orientation. During the experiment, the screen at the front of the apparatus was opaque; the participants could not see the mice. 
Table 1

Mismatch Pairs Differing on the $x$-Dimension (Hole/Bump)

\begin{tabular}{ccc}
\hline \multicolumn{3}{c}{$x$-Pairs } \\
\cline { 2 - 3 } Two Steps & Three Steps & Two Steps \\
\hline 1,3 & 2,5 & 4,6 \\
7,9 & 8,11 & 10,12 \\
13,15 & 14,17 & 16,18 \\
19,21 & 20,23 & 22,24 \\
25,27 & 26,29 & 28,30 \\
31,33 & 32,35 & 34,36 \\
\hline
\end{tabular}

haptic exploration. The participants responded with their left hands, using a button box.

\section{Design and Procedure}

Trials were presented randomly within a block. The participants in the blocked condition completed a block of 36 short (3-sec) ISI trials and a block of 36 long (15-sec) ISI trials. Block order was counterbalanced. The participants in the mixed condition received a single block of 36 short ISI and 36 long ISI trials.

The pairs of mice presented on the 72 trials were identical for all the participants, but the orientation of the first mouse and mouse order on mismatches were counterbalanced across participants. For each ISI, there were 18 matches (presenting the same mouse twice) and 18 mismatches (presenting two different mice). Within each set, 9 trials presented both mice at the same orientation, and 9 trials presented the second mouse rotated by $90^{\circ}$ in depth relative to the first mouse.

All 36 mice were presented on mismatches using $18 x$-dimension (Table 1) and $18 y$-dimension (Table 2) pairings, in which mice differed by two or three steps along the relevant dimension in the shape space. Each mouse was presented four times to each participant: twice on a single match trial when it was presented as both the first and second mouse, and twice as either the first or the second mouse on two separate mismatches.

Half of the mismatches presented participants with the smaller numbered mouse first (e.g., 1 then 3), and half presented the mice in the opposite order. This was counterbalanced across participants. Starting orientation was also counterbalanced across participants, so that, overall, half of both matches and mismatches presented the first mouse at the green orientation, and half presented the first mouse at the red orientation.

On each trial, the experimenter positioned the first mouse, and then triggered a computer to play the words "go now," instructing the participant to move his or her right hand through the aperture. Five seconds after the hand broke the infrared beam, the computer played the words "stop now," indicating that the participant should withdraw his or her hand from the aperture. The experimenter then removed the first mouse and either put it back into the apparatus on matches or replaced it with another mouse on mismatches. Removing and then replacing the mouse on matches ensured that the sounds or movements of the experimenter did not allow the participants to discriminate matches from mismatches. The computer signaled "go now" either $3 \mathrm{sec}$ (short ISI) or $15 \mathrm{sec}$ (long ISI) after the "stop now" signal, indicating that the participant could start to explore the second mouse. The participants decided whether the first and second mice had the same shape and responded with a speeded keypress. A tone provided feedback on accuracy.

Table 2

Mismatch Pairs Differing on the $y$-Dimension (Dip/Ridge)

\begin{tabular}{lrrrrrr}
\hline \multicolumn{7}{c}{$y$-Pairs } \\
\hline Two steps & 1,13 & 2,14 & 3,15 & 4,16 & 5,17 & 6,18 \\
Three steps & 7,25 & 8,26 & 9,27 & 10,28 & 11,29 & 12,30 \\
Two steps & 19,31 & 20,32 & 21,33 & 22,34 & 17,35 & 24,36 \\
\hline
\end{tabular}

Before the start of the experiment, the participants were told to ignore the orientation of the mice and were warned that, on mismatches, the two mice would have very similar shapes. They were shown Mouse 1 and Mouse 31 visually and were then given 10 haptic practice trials with the same ISI as that in the first experimental block in the blocked condition or a mix of ISIs in the mixed condition. These trials were easier than the experimental trials because, for mismatches, the mice were four to five steps apart.

\section{RESULTS}

To test the abstractness and durability of haptic representations, we examined whether people found it harder to detect shape changes when two objects were presented at different orientations and separated further in time. In our previous sequential shape-matching experiments, our analyses focused on matches only (e.g., Craddock \& Lawson, 2009a, 2009b; Lawson, 2009), since mismatches typically presented two very different shapes. Manipulations such as size and orientation were therefore meaningfully interpretable only for matches. In contrast, the objects in the present experiment all shared the same global shape and had well-defined fronts and backs, so we expected similar orientation sensitivity for both matches and mismatches. We therefore chose to follow Norman et al. (2008) in using a signal detection analysis of our results. ${ }^{1}$

We calculated $d^{\prime}$ as a bias-free measure of perceptual sensitivity and $c$ as a measure of response bias (Macmillan $\&$ Creelman, 2005). We replaced cells in which no errors occurred with a value equivalent to half a hit or false alarm (Schooler \& Shiffrin, 2005). We analyzed $d^{\prime}$ and $c$ with a mixed ANOVA using ISI (short or long) and orientation (same or different) as within-participants factors and condition (blocked or mixed) as a between-participants factor.

One participant was replaced in the blocked condition because she made $39 \%$ errors (as compared with a mean for the condition of $20 \%$ ). Four participants were replaced in the mixed condition: 2 because they claimed to have used the sounds of the experimenter moving the mice during the ISI to respond, 1 because she made errors on $42 \%$ of the trials (as compared with a mean for the condition of $16 \%$ ), and 1 because her mean response time (RT) was over $7 \mathrm{sec}$ (condition mean $=3,379 \mathrm{msec}$ ). Thus, in total, 5 participants were replaced with new participants.

In the blocked condition $\left(d^{\prime}=1.84, c=.15\right)$, sensitivity was lower $\left[F(1,62)=5.00, p=.03, \eta_{\mathrm{p}}^{2}=.08\right]$ than in the mixed condition $\left(d^{\prime}=2.05, c=.09\right)$. There was no difference in bias $[F(1,62)=1.01, p=.3]$. There were no significant interactions involving this factor.

At the short ISI $\left(d^{\prime}=1.97, c=.17\right)$, sensitivity was similar $[F(1,62)=0.77, p=.4]$ to that at the long ISI $\left(d^{\prime}=1.91, c=.07\right)$, although there was more bias to respond same $\left[F(1,62)=5.42, p=.02, \eta_{\mathrm{p}}^{2}=.08\right]$.

On same-orientation trials $\left(d^{\prime}=2.02, c=.18\right)$, sensitivity tended to be higher $\left[F(1,62)=3.38, p=.07, \eta_{\mathrm{p}}^{2}=\right.$ $.05]$, and the bias to respond same was greater $[F(1,62)=$ $\left.12.19, p=.001, \eta_{\mathrm{p}}^{2}=.16\right]$ than on different-orientation trials $\left(d^{\prime}=1.87, c=.06\right)$. 
A

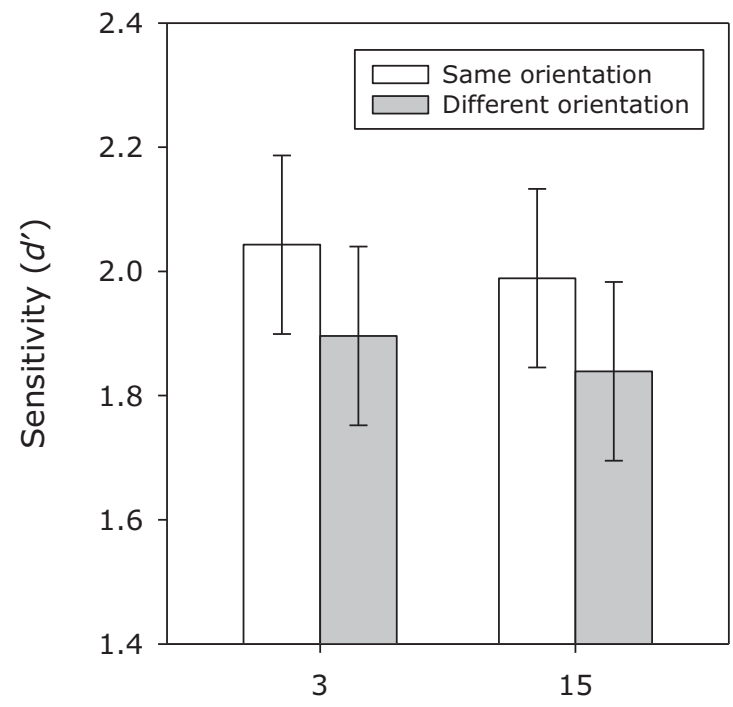

Interstimulus Interval (sec)
B

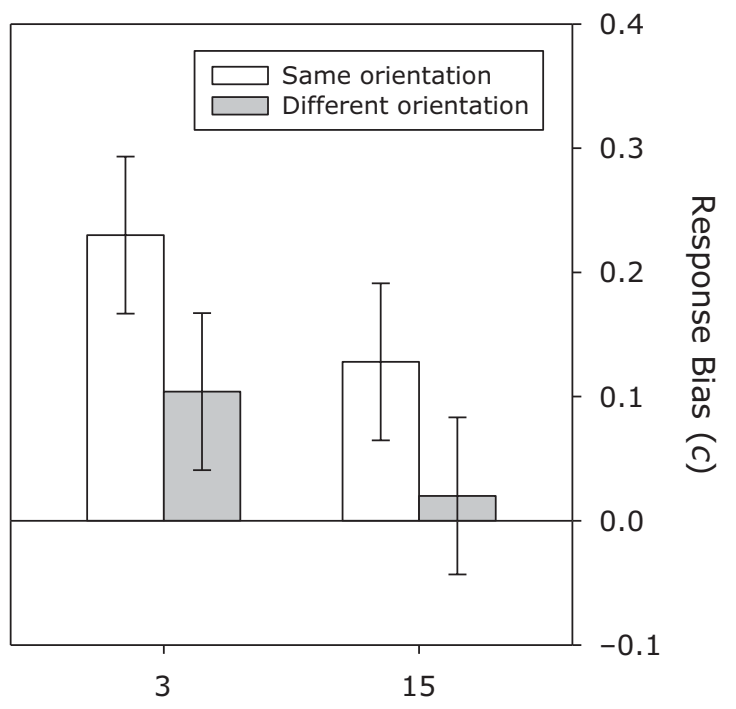

Interstimulus Interval (sec)

Figure 4. Sensitivity (A) and response bias (B) for same-orientation and different-orientation trials at the two interstimulus intervals (ISIs). Error bars represent $95 \%$ within-participants confidence intervals calculated using the error term of the ISI $\times$ orientation interaction (Jarmasz \& Hollands, 2009; Loftus \& Masson, 1994).

Critically, there was no interaction between orientation and ISI for sensitivity $[F(1,62)=0.001, p>.9]$ or bias $[F(1,62)=0.08, p=.8$; see Figure 4].

\section{DISCUSSION}

We found no evidence of a change in sensitivity at the long 15-sec ISI, as compared with the short 3-sec ISI, although there was a reduction in response bias. Our results are consistent with those of Kiphart et al. (1992), who found that haptic matching performance was maintained between 5 and $15 \mathrm{sec}$. In contrast, Woods et al. (2004) reported that performance declined between 0 and $15 \mathrm{sec}$. This difference may be due to superior performance at very short delays being mediated by a transient sensory representation.

Most interesting, our results do not replicate those of Norman et al. (2008), who had naturalistic stimuli most similar to the mice presented in this study. They suggested that people's ability to detect subtle changes in 3-D object shape on a haptic matching task improved as ISI increased, on the basis of their finding that hit rates increased and response bias decreased at $15-\mathrm{sec}$, as compared with 3-sec, ISIs. However, the reduction in bias implies that the improvement in hit rate was offset by an increase in the number of false alarms, since they reported a significant effect of ISI only on hit rate, not on sensitivity. Furthermore, we found a similar pattern of results here: Our analysis showed that although there was an increase in the hit rate at the 15-sec ISI, there was a similar-sized increase in false alarms and, therefore, sensitivity did not improve. Thus, Norman et al.'s report of improvements in performance as ISI increased may have been due to a biased performance measure; their results are actually consistent with both our findings and those of Kiphart et al. (1992).

Although we found a trend in the expected direction for higher sensitivity on same-orientation trials, this benefit was not as clear as we had previously observed in similar tasks (e.g., Lawson, 2009). Nevertheless, this trend and the increased bias to respond same on same-orientation trials also suggests that orientation was stored. Orientation sensitivity may have been relatively weak here because the stimuli all had similar global shapes and the same front-back main axis of elongation that clearly defined their orientation. The ease of defining orientation for these stimuli, as compared with the large, heterogeneous set of models of familiar, nameable objects that we used previously (Lawson, 2009), may have made it easier to learn to compensate for orientation changes within the study.

We found no evidence that the same-orientation benefit weakened at longer ISIs (see Figure 4). This was contrary to our predictions based on findings in visual object recognition (Ellis \& Allport, 1986; Ellis et al., 1989; Lawson \& Humphreys, 1996). Instead, these results suggest that even after a delay of $15 \mathrm{sec}$, haptically acquired information about 3-D objects is maintained in orientationsensitive perceptual representations, rather than being transferred to more abstract, orientation-invariant semantic or name representations. This is consistent with our finding of long-term maintenance of orientation information for haptically explored familiar objects (Craddock \& Lawson, 2008) and our suggestion that haptics would maintain initial input with relatively little abstraction for a reasonable period of time. Our results suggest that, when 
the right key is chosen from a bunch by touch, there is no need to rush, but it would be easier if the keys were all pointing in the same direction!

\section{AUTHOR NOTE}

This research was supported by an Engineering and Physical Sciences Research Council DTA studentship to the first author and by a fellowship from the Economic and Social Research Council (RES-000-27-0162) to the second author. Correspondence concerning this article should be addressed to M. Craddock, School of Psychology, University of Liverpool, Eleanor Rathbone Building, Bedford Street South, Liverpool L69 7ZA, England (e-mail: m.craddock@liv.ac.uk).

\section{REFERENCES}

Amedi, A., Jacobson, G., Hendler, T., Malach, R., \& Zohary, E. (2002). Convergence of visual and tactile shape processing in the human lateral occipital complex. Cerebral Cortex, 12, 1202-1212. doi:10.1093/cercor/12.11.1202

Amedi, A., Malach, R., Hendler, T., Peled, S., \& Zohary, E. (2001). Visuo-haptic object-related activation in the ventral visual pathway. Nature Neuroscience, 4, 324-330. doi:10.1038/85201

Craddock, M., \& Lawson, R. (2008). Repetition priming and the haptic recognition of familiar and unfamiliar objects. Perception \& Psychophysics, 70, 1350-1365. doi:10.3758/PP.70.7.1350

Craddock, M., \& Lawson, R. (2009a). The effects of size changes on haptic object recognition. Attention, Perception, \& Psychophysics, 71, 910-923. doi:10.3758/APP.71.4.910

Craddock, M., \& Lawson, R. (2009b). Size-sensitive perceptual representations underlie visual and haptic object recognition. PLoS ONE, 4, e8009. doi:10.1371/journal.pone.0008009

Ellis, R., \& Allport, D. A. (1986). Multiple levels of representation for visual objects: A behavioural study. In A. G. Cohn \& J. R. Thomas (Eds.), Artificial intelligence and its applications (pp. 245-257). Chichester, U.K.: Wiley.

Ellis, R., Allport, D. A., Humphreys, G. W., \& Collis, J. (1989). Varieties of object constancy. Quarterly Journal of Experimental Psychology, 41A, 775-796.

Gallace, A., Tan, H. Z., Haggard, P., \& Spence, C. (2008). Short term memory for tactile stimuli. Brain Research, 1190, 132-142. doi:10.1016/j.brainres.2007.11.014

Gilson, E. Q., \& BAdDELEy, A. D. (1969). Tactile short-term memory. Quarterly Journal of Experimental Psychology, 21, 180-184. doi:10.1080/14640746908400211

Humphrey, G. K., \& LupKer, S. J. (1993). Codes and operations in picture matching. Psychological Research, 55, 237-247.

JARMASZ, J., \& HollandS, J. G. (2009). Confidence intervals in repeatedmeasures designs: The number of observations principle. Canadian Journal of Experimental Psychology, 63, 124-138. doi:10.1037/ a0014164

Kiphart, M. J., Hughes, J. L., Simmons, J. P., \& Cross, H. A. (1992). Short-term haptic memory for complex objects. Bulletin of the Psychonomic Society, 30, 212-214.

Klatzky, R. L., Lederman, S. J., \& Metzger, V. A. (1985). Identifying objects by touch: An "expert system." Perception \& Psychophysics, 37, 299-302.
Lacey, S., Peters, A., \& Sathian, K. (2007). Cross-modal object recognition is viewpoint-independent. PLoS ONE, 2, e890. doi:10.1371/ journal.pone. 0000890

LaWson, R. (1999). Achieving visual object constancy across plane rotation and depth rotation. Acta Psychologica, 102, 221-245. doi:10.1016/ S0001-6918(98)00052-3

LAwson, R. (2009). A comparison of the effects of depth rotation on visual and haptic three-dimensional object recognition. Journal of Experimental Psychology: Human Perception \& Performance, 35, 911-930. doi:10.1037/a0015025

Lawson, R., \& Humphreys, G. (1996). View specificity in object processing: Evidence from picture matching. Journal of Experimental Psychology: Human Perception \& Performance, 22, 395-416. doi:10.1037/0096-1523.22.2.395

Loftus, G. R., \& MAsson, M. E. J. (1994). Using confidence intervals in within-subject designs. Psychonomic Bulletin \& Review, 1, 476-490.

Macmillan, N. A., \& Creelman, C. D. (2005). Detection theory: A user's guide. Mahwah, NJ: Erlbaum.

Miles, C., \& Borthwick, H. (1996). Tactile short-term memory revisited. Memory, 4, 655-668.

MiLlar, S. (1974). Tactile short-term memory by blind and sighted children. British Journal of Psychology, 65, 253-263.

Miquée, A., Xerri, C., Rainville, C., Anton, J.-L., Nazarian, B., Roth, M., \& Zennou-Azogui, Y. (2008). Neuronal substrates of haptic shape encoding and matching: A functional magnetic resonance imaging study. Neuroscience, 152, 29-39. doi:10.1016/ j.neuroscience.2007.12.021

Newell, F. N., ERnst, M. O., Tuan, B. S., \& Bülthoff, H. H. (2001). Viewpoint dependence in visual and haptic object recognition. Psychological Science, 12, 37-42. doi:10.1111/1467-9280.00307

Norman, J. F., Clayton, A. M., Norman, H. F., \& Crabtree, C. E. (2008). Learning to perceive differences in solid shape through vision and touch. Perception, 37, 185-196. doi:10.1068/p5679

Schooler, L. J., \& SHIFFrin, R. M. (2005). Efficiently measuring recognition performance with sparse data. Behavior Research Methods, 37, 3-10.

Sullivan, E. V., \& Turvey, M. T. (1972). Short-term retention of tactile stimulation. Quarterly Journal of Experimental Psychology, 24, 253261. doi:10.1080/14640747208400278

TARR, M. J., \& CHENG, Y. D. (2003). Learning to see faces and objects. Trends in Cognitive Sciences, 7, 23-30. doi:10.1016/S1364-6613(02) 00010-4.

Woods, A. T., O'Modhrain, S., \& Newell, F. N. (2004). The effect of temporal delay and spatial differences on cross-modal object recognition. Cognitive, Affective, \& Behavioral Neuroscience, 4, 260-269. doi:10.3758/CABN.4.2.260

\section{NOTE}

1. We also performed analyses of RTs and error rates, as in our previous articles. These analyses revealed orientation sensitivity, with significant speed and accuracy advantages on same-orientation matches.

(Manuscript received December 14, 2009; revision accepted for publication June 1, 2010.) 